Le Signe et la consigne. Essai sur la genèse de l'œuvre en régime naturaliste. Zola, dir. Philippe Hamon, Genève, Droz, 2009, 320 p.

\title{
Céline Grenaud
}

\section{(2) OpenEdition}

Journals

Édition électronique

URL : http://journals.openedition.org/genesis/651

DOI : 10.4000/genesis. 651

ISSN : 2268-1590

Éditeur :

Presses universitaires de Paris Sorbonne (PUPS), Société internationale de génétique artistique littéraire et scientifique (SIGALES)

\section{Édition imprimée}

Date de publication : 30 octobre 2011

Pagination : 190-191

ISBN : 978-2-84050-804-5

ISSN : $1167-5101$

\section{Référence électronique}

Céline Grenaud, «Le Signe et la consigne. Essai sur la genèse de l'œuvre en régime naturaliste. Zola, dir. Philippe Hamon, Genève, Droz, 2009, 320 p. », Genesis [En ligne], 33 | 2011, mis en ligne le 23 octobre 2013, consulté le 22 septembre 2020. URL : http://journals.openedition.org/genesis/651 DOI : https://doi.org/10.4000/genesis.651 
sensibilité artistique de Valéry avant 1912 et se trouve-t-il conduit à accorder une place décisive à « la musique intime du moi », étrange énergie jusqu'ici peu observée semble-t-il dans les études valéryennes.

C'est alors un passionnant portrait d'écrivain à l'ouvrage qui se dessine, largement « autoanalysé » par Valéry lui-même, où sensibilité et intelligence sont aux prises et forment un thyrse complexe. Brian Stimpson met en lumière les rythmes de la création valéryenne où se succèdent un " temps de latence créatrice » durant lequel « l'ignorance même est dynamique », et un temps de " découverte réfléchie » où la question $\mathrm{du}$ 《faire » se trouve posée à la fois sous l'angle du quoi et du comment. Il apparaît que l'essentiel du travail consiste en rapprochements et en combinaisons. L'écriture de Valéry présente un caractère singulièrement évolutif et tâtonnant. Brian Stimpson montre comment le poème sort de sa gangue, se dégage de ses ratures, la manière dont la pensée se clarifie : le travail de la langue est travail de l'esprit.

$\mathrm{Au}$ commencement est un potentiel dont il faudra débrouiller une forme, des possibles d'où extraire des nécessités, une matière germinale pareille à quelque « soupe primordiale ». Paul Valéry, à maints égards, est un explorateur et, avant la lettre, son propre généticien : il ne quitte pas des yeux le mûrissement de son travail et en développe l'analyse. N'est-ce pas là, après tout, ce qui dans l'écriture l'intéresse le plus : y voir l'esprit fonctionner ? Comme l'observe Brian Stimpson, « l'écrire des Cahiers et la pratique des manuscrits de la poésie sont intimement imbriqués ».

Pour emboîter le pas sans faillir à un pareil explorateur, il fallait un soin et un souffle de spéléologue : c'est avec autant de prudence que de qualité d'attention et de méticulosité critique que Brian Stimpson offre ces nouvelles entrées dans le temps de l'écriture et dans le faire du poète, ce faire vorace qui lui-même interroge, brasse et assimile les matériaux les plus divers. Il a su ne pas figer Valéry à partir du texte connu et maintes fois déjà commenté ; il est revenu aux sources au lieu de procéder à rebours et d'effacer du même coup ces hésitations et ces glissements imperceptibles qui sont la vie même de l'écrit.

Ainsi ce livre aussi riche que sérieux autorise-t-il de troublantes retrouvailles. Émouvante en effet est la réalité prise par la voix de Valéry dans cette étude : par le poème, la pensée et les détours complexes de l'écrit, mais aussi par des lettres intimes (telles celles écrites à sa femme depuis Perros-Guirec en juillet 1913)... Le tout confère à Valéry une présence rare dans un livre de cette espèce. Valéry au travail, ciselant le son et le sens, et Valéry sensible, sensoriel, frissonnant parfois, n'y font qu'un.

Le Signe et la consigne. Essai sur la genèse de l'ouvre en régime naturaliste. Zola, dir. Philippe Hamon, Genève, Droz, 2009, 320 p.

\section{Compte rendu par Céline Grenaud}

« Nos œuvres en gestation échappent absolument à notre volonté », écrivait Zola à Huysmans, dans une lettre du 20 mai 1884. De fait, la question se pose de savoir si la création coïncide avec la mise en œuvre de processus parfaitement maîtrisés, facilement repérables et systématiquement appliqués ou bien si elle échappe à la conscience et reste placée, en définitive, sous le signe du mystère et de la géniale improvisation. L'ouvrage collectif réalisé sous la direction de Philippe Hamon permet de nuancer la réponse. Il fait émerger, d'une manière remarquablement synthétique, les principes structurants de la méthode zolienne, tels qu'ils se donnent à lire dans l'ensemble des dossiers préparatoires. Mais il encourage aussi à décrypter le métadiscours d'un écrivain qui mêle constamment l'interrogation à l'invention et ne cesse, à la faveur d'un « soliloque programmatif » extrêmement dense, de dialoguer avec lui-même, d'ancrer son propos dans les problématiques contemporaines, en somme de poser l'écriture comme un acte complexe, impossible à circonscrire dans les limites du prévisible.

L'ouvrage invite à confronter les avant-textes zoliens aux catégories de la rhétorique classique : inventio, dispositio, elocutio, memoria et actio. La création ne se réduisant pas à la succession rectiligne de séquences fixes et exclusives les unes des autres, ce cadrage est rendu opérationnel par la reconnaissance d'un enchevêtrement permanent des opérations. Une telle démarche permet de mesurer l'efficacité des protocoles activés par l'auteur dans la phase prérédactionnelle, enrichis de dossier préparatoire en dossier préparatoire et nourris par une série de consignes plus ou moins récurrentes. L'un des principaux mérites de cet essai est d'aboutir ainsi à la compréhension globale des mécanismes impliqués dans la genèse des romans naturalistes. Il parvient à mêler une approche macroscopique destinée à éclairer la totalité d'une œuvre et une étude microscopique tenant compte des infimes détails grâce auxquels l'écriture zolienne signe sa différence.

Dans un premier chapitre, Alain Pagès s'attarde sur les différents niveaux de l'inventio : la question initiale, d'abord - Nana envisagé comme le «poème des désirs du mâle » ou Au Bonheur des dames comme celui « de l'activité moderne »-, puis surtout la recherche documentaire, essentielle dans une vision du monde où la logique doit prévaloir sur l'imagination. Les avant-textes témoignent de ce que la collecte des informations débouche sur l'obsession du classement et induit fréquemment le recours à des experts (Pol Lefèvre pour La Bête humaine, Alfred Roll pour Germinal, etc.) ou à des amis et correspondants avisés (Paul Alexis, Henry Céard). Alain Pagès envisage aussi la mise en scène du dilemme et la crainte lancinante de mal faire, telle qu'elle est trahie par les structures de l'alternative ou de l'épanorthose. 
OlivierLumbrosometensuiteenévidence les modèles qui permettent de caractériser la composition des romans zoliens. Leur « mise en ordre syntagmatique », d'une part, suppose la distribution du scénario dans l'Ébauche (avec parfois une programmation précoce des chapitres), puis dans les Plans détaillés. Leur « mise en ordre hiérarchique », d'autre part, correspond à la distinction des plans principaux et secondaires, à la distribution des valeurs positives ou négatives et à la désignation des centralités actantielles (la figure centrale doit « rayonner »), spatiales et narratives. Ce système, doté d'une relative souplesse, autorise la polyhiérarchisation et se combine régulièrement avec des jeux d'ombre et de lumière.

Dans un troisième chapitre, Philippe Hamon s'attache à décoder les principes de l'elocutio et, malgré la circonspection de Zola en la matière, à mettre en relief les consignes associées à la caractérisation de son style. Les dossiers préparatoires attestent, ne serait-ce que d'un point de vue graphique (guillemets, soulignement...), du soin avec lequel l'écrivain choisit ses mots. Ceuxci, tour à tour, sont investis d'une fonction narrative particulière (le choix d'un terme populaire n'est pas anodin), apparaissent plus ou moins ostensiblement motivés (c'est le cas de nombreux noms propres) ou encore résultent d'un parti pris déterminant en terme d'oralité (la parole présente souvent un aspect ethnographique et sociologique).

Le quatrième chapitre est consacré au métadiscours mémoriel. Chantal Pierre-Gnassounou analyse les traces de l'intratextualité (les dossiers sont émaillés de souvenirs procédant de l'œuvre déjà réalisée) et celles de l'intertextualité (l'auteur alterne entre la tentation de l'imitation et le désir de dissociation). Elle étudie aussi la cohérence des données citationnelles et du système actantiel, puis éclaire le jeu des variations, visiblement ajusté à la volonté d'éviter les répétitions. Les références autobiographiques, quoique relativement rares dans les dossiers, conduisent enfin à sonder la mémoire de soi.
Philippe Hamon envisage dans le cinquième chapitre l'ultime séquence du paradigme rhétorique. L'actio se manifeste par le souci des rythmes : rythme quasi biologique de l'auteur en plein acte d'écriture, rythme de la série cadencée par des romans forts et faibles, ou encore rythme stylistique, tel qu'il est programmé dans le dossier de L'Assommoir (Zola prévoit un «style à toute volée »). Il importe aussi de prendre en considération les gesticulations graphiques (biffures, ratures, soulignements...) et celles, plus subtiles, qui impliquent la mise en œuvre des fonctions conative et émotive.

En guise de conclusion, Henri Mitterand propose une réflexion sur « le modèle et la liberté ». Ce faisant, il soulève plusieurs des questions essentielles afférentes à l'écriture en acte. Quelles places respectives faut-il accorder à la topique et à la spontanéité ? Selon quelles modalités s'effectue le passage d'un discours programmatif infaillible sur le plan méthodologique à une fiction narrative beaucoup plus libre, placée sous le signe de la mythographie et parfois même du lyrisme ? Ou encore, comment évaluer les poids de l'ethos et du pathos, c'est-à-dire les qualités morales de l'auteur et les émotions suscitées chez un lecteur potentiel, auquel aujourd'hui nous pouvons d'ailleurs nous identifier ? Autant d'interrogations qui confirment la pertinence d'une approche génétique de l'œuvre zolienne et, au-delà, de la production naturaliste.

Critique génétique et textologie: épistémologie et théorie, dir. T. V. Balachova, E. D. Galtsova, et al., Moscou, IMLI RAN, 2008, 272 p. (Tekstologija i geneticheskaja kritika: Obwie problemy, teoreticheskie perspektivy - Текстология и генетическая критика: общие проблемы, теоретические перспективы). [en russe]

\section{Compte rendu par Valentina Chepiga}

Il s'agit d'un recueil d'articles paru à la suite du colloque franco-russe à l'Institut de Littérature mondiale de l'Académie des sciences de Russie qui a eu lieu les 25 et 26 septembre 2000 dans le cadre du programme de coopération entre l'IMLI de l'Académie des sciences de Russie et l'ITEM du CNRS. Il est constitué d'articles de chercheurs français et russes qui se consacrent, pour les domaines de la littérature, de la philologie et de l'histoire de la culture, à l'étude des problèmes théoriques de la textologie et de la critique génétique, à son application pratique ainsi qu'à la méthodologie d'analyse, que ce soit d'œuvres littéraires et philosophiques des XIXe et $\mathrm{XX}^{\mathrm{e}}$ siècles, ou de différentes pratiques de l'écriture autobiographique. Le recueil réunit trois grands volets : " Théorie », « Euvres des XIX ${ }^{\mathrm{e}}-\mathrm{XX}^{\mathrm{e}}$ siècles à la lumière de la textologie et de la critique génétique » et «Autobiographie et genèse ».

Dans l'introduction, " Le canon insaisissable », T. V. Balachova ouvre ce recueil par une réflexion sur la complémentarité et la divergence des méthodes respectives de la textologie et de la critique génétique. Ainsi, en présentant brièvement l'histoire des deux écoles, elle aborde les problèmes de définition de l'avant-texte, elle met en relief les oppositions des deux approches sur le statut du texte édité dans l'œuvre de l'écrivain et met l'accent sur le fait que les deux écoles sont en dialogue permanent. S'ensuit logiquement le premier article, « La critique génétique française pour la textologie russe », par A. L. Grichounine, qui ouvre le volet théorique du recueil et apporte une importante contribution à l'histoire de la critique génétique russe, celle-ci pouvant être considérée comme faisant partie de la textologie : les noms de Potebnia, Vinokur, Pixanov et d'autres philologues russes qui ont eu le mérite de fonder les bases théoriques de l'approche génétique russe apparaissent à côté des noms des théoriciens français en génétique du texte. Cet article va de pair avec la contribution de E. E. Dmitrieva, « Glossaire croisé : critique génétique et textologie», qui s'appuie sur le 